\title{
Adubação nitrogenada e potássica de cafeeiro fertirrigado na fase de formação, em plantio adensado
}

\author{
Fabrício Moreira Sobreira(1), Rubens José Guimarães(2), Alberto Colombo(3), Myriane Stella Scalco ${ }^{(2)}$ \\ e Janice Guedes Carvalho(4)
}

\begin{abstract}
(1)Universidade Federal de Viçosa, Departamento de Fitotecnia, Avenida P. H. Rolfs, s/no, CEP 36570-000 Viçosa, MG. E-mail: sobreirafm@bol.com.br (2)Universidade Federal de Lavras (Ufla), Departamento de Agricultura, Caixa Postal 37, CEP 37200-000 Lavras, MG. E-mail: rubensjg@dag.ufla.br msscalco@dag.ufla.br (3)Ufla, Departamento de Engenharia, E-mail: acolombo@deg.ufla.br ${ }^{(4)}$ Ufla, Departamento de Ciência do Solo, E-mail: janicegc@dcs.ufla.br
\end{abstract}

Resumo - O objetivo deste trabalho foi determinar o parcelamento e a dose de nitrogênio e potássio mais adequados para o primeiro e segundo anos de formação do cafeeiro (Coffea arabica) fertirrigado por gotejamento, no sul de Minas Gerais. Dois experimentos foram instalados simultaneamente com plantio adensado (6.666 plantas ha $\left.{ }^{-1}\right)$ : em um, a adubação foi realizada em quatro aplicações ao ano e, no outro, em 12 aplicações. Utilizou-se o delineamento em blocos ao acaso com cinco tratamentos e quatro repetições em ambos os experimentos. Os tratamentos foram constituídos por doses, aplicadas via fertirrigação, equivalentes a 70,100, 130, 160 e 190\% da recomendada para $\mathrm{Ne} \mathrm{K}_{2} \mathrm{O}$, para o cultivo em sequeiro. Foi realizado tratamento controle com cultivo em sequeiro e adubação com a dose padrão: $100 \%$ da recomendada. No primeiro e segundo anos, em ambos os tipos de parcelamento, não houve diferença significativa entre as doses, quanto ao crescimento do cafeeiro. O parcelamento em 12 aplicações é mais adequado para adubação de primeiro e segundo anos pós-plantio. Cafeeiros em formação fertirrigados por gotejamento apresentam maior crescimento e menor demanda por adubação com $\mathrm{N}$ e $\mathrm{K}$ do que os cultivados em sequeiro.

Termos para indexação: Coffea arabica, demanda nutricional, irrigação por gotejamento, parcelamento da adubação, sequeiro.

\section{Nitrogen and potassium fertigation in coffee at the formation phase, with high plant density}

\begin{abstract}
The objective of this study was to evaluate the most adequate parceling and dose of nitrogen and potassium for the first and second years of coffee shrub (Coffea arabica) formation, under a drip fertigation system, in southern Minas Gerais, Brazil. Two experiments with high plant density (6.666 plants ha $\left.\mathrm{a}^{-1}\right)$ were carried out simultaneously: in one of them, the fertilization was done in 4 applications yearly, and in the other one, in 12 applications. A randomized block design was used with five treatments and four replicates in both experiments. Treatments consisted of $\mathrm{N}$ and $\mathrm{K}_{2} \mathrm{O}$ doses applied via fertigation: 70, 100, 130, 160 and 190\% from the $\mathrm{N}$ and $\mathrm{K}_{2} \mathrm{O}$ recommended doses for the rainfed cropping. A control treatment was carried out in rainfed conditions and fertilized with the standard dose (100\%). In the first and second years, in both parceling types, there were no significant differences among doses regarding coffee shrub vegetative growth. Parceling into 12 applications is more suitable for fertilization at both first and second years after planting. Fertigated coffee shrubs at the formation phase have higher growth and demand less nitrogen and potassium fertilization than the ones in rainfed conditions.
\end{abstract}

Index terms: Coffea arabica, nutritional demand, drip irrigation, split fertilization, rainfed.

\section{Introdução}

Na cafeicultura (Coffea arabica e C. canephora), a ocorrência de veranicos em fases fenológicas críticas da cultura provoca consideráveis perdas de produtividade e qualidade dos grãos (DaMatta \& Ramalho, 2006; DaMatta et al., 2007; Silva et al., 2008b). Para evitar perdas e potencializar o ganho produtivo, a irrigação por gotejamento, muitas vezes associada à fertirrigação, tem sido adotada de forma expressiva pelos cafeicultores, inclusive em regiões como o sul de Minas, onde pesquisadores ainda discutem sua viabilidade (Silva et al., 2003; 2008a; Gomes et al., 2007). 
Comparada ao sistema convencional de adubação, a fertirrigação possibilita aumentar a produtividade e melhorar a qualidade dos frutos do cafeeiro, reduz custos com mão de obra na adubação e aumenta a eficiência no manejo do solo (Silva et al., 2003; Gomes et al., 2007; Carvalho et al., 2010; Pereira et al., 2010). Contudo, os benefícios da técnica são limitados em razão da escassez de informações científicas específicas para o cafeeiro, sobretudo quanto a doses e número de parcelamentos necessários no ano. A fertirrigação na cafeicultura vem sendo discutida com base em trabalhos relacionados à irrigação (Silva et al., 2003, 2008b; Gomes et al., 2007), à adubação líquida (Fagundes, 2006) e à fertirrigação (Karasawa et al., 2002; Nazareno et al., 2003; Costa, 2005; Rezende et al., 2010).

Para o cafeeiro cultivado em sequeiro, as principais regiões produtoras estabeleceram, após anos de pesquisa, as doses e parcelamentos de $\mathrm{N} \mathrm{e}_{2} \mathrm{O}$ mais adequadas ao período de formação da lavoura (Guimarães et al., 1999). Entretanto, para o cafeeiro fertirrigado, tais informações ainda não estão definidas.

O objetivo deste trabalho foi determinar o parcelamento e a dose de nitrogênio e potássio mais adequados para o primeiro e segundo anos de formação do cafeeiro (C. arabica) fertirrigado por gotejamento, no sul de Minas Gerais.

\section{Material e Métodos}

Dois experimentos foram conduzidos em Lavras, MG ( $21^{\circ} 14^{\prime} 06^{\prime \prime} \mathrm{S}$ e $45^{\circ} 00^{\prime} 00^{\prime \prime} \mathrm{W}$, altitude de $\left.910 \mathrm{~m}\right)$, no Setor de Cafeicultura do Departamento de Agricultura da Universidade Federal de Lavras (Ufla), de 2007 a 2009. O solo da área experimental é um Latossolo Vermelho-Escuro distroférrico, de textura argilosa a muito argilosa, e suas características químicas, à profundidade de 0 a $20 \mathrm{~cm}$, estão apresentadas na Tabela 1.

Em um dos experimentos, o parcelamento de $\mathrm{N}$ e $\mathrm{K}$ foi realizado em quatro aplicações (P4) ao ano, na época de chuvas da região - novembro, dezembro, janeiro e fevereiro - e, no outro experimento em 12 aplicações (P12) ao ano. Os experimentos foram implantados simultaneamente em áreas próximas, e o intervalo entre as aplicações foi de 30 dias. Nos dois experimentos, foi utilizado o delineamento em blocos ao acaso, com cinco níveis de adubação como tratamentos, aplicados via fertirrigação, com doses equivalentes de 70, 100, 130,
160 e $190 \%$ da recomendada para adubação com N e $\mathrm{K}_{2} \mathrm{O}$ em cultivos de sequeiro (Guimarães et al., 1999). Foram utilizadas quatro repetições com dez plantas por parcela. O crescimento das plantas foi avaliado em seis épocas, no primeiro ano, e quatro épocas, no segundo. Como parcela útil, foram consideradas as oito plantas centrais. Para cada linha de tratamento, foram implantadas duas linhas de bordadura: uma de cada lado.

Comodose-padrão(100\%), foi adotada a recomendada por Guimarães et al. (1999), para a cultura do cafeeiro em sequeiro na fase de formação e apresentando teor de $\mathrm{K}$ no solo entre $120-200 \mathrm{mg} \mathrm{dm}^{-3}$. A dose-padrão recomendada foi de $10 \mathrm{~g}$ de $\mathrm{K}_{2} \mathrm{O}$ e $40 \mathrm{~g}$ de $\mathrm{N}$ por planta por ano, para o primeiro ano de formação da lavoura, e de $20 \mathrm{~g}$ de $\mathrm{K}_{2} \mathrm{O}$ e $80 \mathrm{~g}$ de $\mathrm{N}$ por planta por ano, para o segundo. Guimarães et al. (1999) recomendaram as doses de fertilizantes, na fase de formação, por planta ao invés de por área, por considerar que não há competição entre as plantas nessa fase. Em cada aplicação, utilizou-se a mesma quantidade de $\mathrm{Ne} \mathrm{K}_{2} \mathrm{O}$, medida em gramas. Ao final das aplicações, ambos os

Tabela 1. Caracterização química do solo da área à profundidade de $0-20 \mathrm{~cm}$, antes da aplicação dos tratamentos.

\begin{tabular}{|c|c|}
\hline Característica $^{(1)}$ & Resultado \\
\hline $\mathrm{pH}\left(\mathrm{H}_{2} \mathrm{O}\right)$ & 6,7 \\
\hline $\mathrm{P}\left(\mathrm{mg} \mathrm{dm} \mathrm{m}^{-3}\right)$ & 15,4 \\
\hline $\mathrm{K}\left(\mathrm{mg} \mathrm{dm}^{-3}\right)$ & 137 \\
\hline $\mathrm{Ca}^{2+}\left(\mathrm{cmol}_{\mathrm{c}} \mathrm{dm}^{-3}\right)$ & 4,4 \\
\hline $\mathrm{Mg}^{2+}\left(\mathrm{cmol}_{\mathrm{c}} \mathrm{dm}^{-3}\right)$ & 1,5 \\
\hline $\mathrm{Al}^{3+}\left(\mathrm{cmol}_{\mathrm{c}} \mathrm{dm}^{-3}\right)$ & 0,0 \\
\hline $\mathrm{H}+\mathrm{Al}\left(\mathrm{cmol}_{\mathrm{c}} \mathrm{dm}^{-3}\right)$ & 2,1 \\
\hline Soma de bases $\left(\mathrm{cmol}_{\mathrm{c}} \mathrm{dm}^{-3}\right)$ & 6,3 \\
\hline CTC $\left(\mathrm{cmol}_{\mathrm{c}} \mathrm{dm}^{-3}\right)$ & 6,3 \\
\hline CTC, $\mathrm{pH} 7,0\left(\mathrm{cmol}_{\mathrm{c}} \mathrm{dm}^{-3}\right)$ & 8,3 \\
\hline Saturação por bases (\%) & 74,9 \\
\hline Satururação por alumínio (\%) & 0 \\
\hline Matéria orgânica $\left(\right.$ dag kg-1 $\left.^{-1}\right)$ & 4,3 \\
\hline P remanescente $(\mathrm{P}-\mathrm{rem})\left(\mathrm{mg} \mathrm{L}^{-1}\right)$ & 7,7 \\
\hline $\mathrm{Zn}\left(\mathrm{mg} \mathrm{dm}^{-3}\right)$ & 5,1 \\
\hline $\mathrm{Fe}\left(\mathrm{mg} \mathrm{dm}^{-3}\right)$ & 76,7 \\
\hline $\operatorname{Mn}\left(\mathrm{mg} \mathrm{dm}^{-3}\right)$ & 22,5 \\
\hline $\mathrm{Cu}\left(\mathrm{mg} \mathrm{dm}^{-3}\right)$ & 3,7 \\
\hline $\mathrm{B}\left(\mathrm{mg} \mathrm{dm}^{-3}\right)$ & 0,2 \\
\hline $\mathrm{S}\left(\mathrm{mg} \mathrm{dm}^{-3}\right)$ & 38,21 \\
\hline
\end{tabular}

${ }^{(1)} \mathrm{P}, \mathrm{K}, \mathrm{Fe}, \mathrm{Zn}, \mathrm{Mn}, \mathrm{Cu}$, extrator Mehlich 1; Ca, Mg, Al, extractor $\mathrm{KCl} 1$ mol L-1 $\mathrm{H}+\mathrm{Al}$, extrator SMP; B, extrator água quente; $\mathrm{S}$, extrator fosfato monocálcico em ácido acético; $\mathrm{MO}$, oxidação $-\mathrm{Na}_{2} \mathrm{Cr}_{2} \mathrm{O}_{7} 4 \mathrm{~N}+\mathrm{H}_{2} \mathrm{SO}_{4} 10 \mathrm{~N}$. 
experimentos ( $\mathrm{P} 2$ e $\mathrm{P} 4)$ receberam a mesma quantidade de $\mathrm{N}$ e $\mathrm{K}_{2} \mathrm{O}$.

A recomendação de adubação foi feita conforme Guimarães etal. (1999), com base no resultado da análise da amostra de solo. O fósforo - superfosfato simples - foi aplicado no plantio da cultura, (abril/2007) em dose única (65 g por cova), e não foi mais utilizado em adubações no período do presente estudo. Os adubos utilizados para o fornecimento de $\mathrm{N} \mathrm{e}_{2} \mathrm{O}$ foram: ureia pecuária $(45 \%$ de $\mathrm{N})$, por sua pureza, solubilidade e preços concorrentes com a ureia fertilizante; e o nitrato de potássio $\left(13 \%\right.$ de $\mathrm{N}$ e $44 \%$ de $\left.\mathrm{K}_{2} \mathrm{O}\right)$. Em razão das características do solo (Tabela 1), optou-se pelo fornecimento de micronutrientes via foliar. $\mathrm{O}$ teor foliar de nutrientes foi monitorado a cada 60 dias e, quando necessário, foi aplicado um produto comercial com $6 \%$ de $\mathrm{Z}, 3 \%$ de $\mathrm{B}, 2 \%$ de $\mathrm{Mn}, 10 \%$ de $\mathrm{Cu}, 10 \%$ de $\mathrm{S}, 1 \%$ de $\mathrm{Mg}$ e $10 \%$ de $\mathrm{K}_{2} \mathrm{O}$, por pulverização via foliar, com volume de calda de 100 a $120 \mathrm{~L} \mathrm{ha}^{-1}$.

Ambos os parcelamentos (P4 e P12) iniciaram-se em 28 de novembro de 2007. No primeiro e segundo anos de adubação, o P4 foi encerrado no final de fevereiro, e o P12 ao final de outubro. Instalou-se, em cada experimento, um tratamento controle cultivado em sequeiro e adubado com a dose-padrão (100\%) recomendada para essa forma de cultivo. Nesse tratamento, o parcelamento da adubação foi realizado em quatro aplicações na época de chuvas - novembro, dezembro, janeiro, fevereiro, também com intervalos de 30 dias entre as aplicações.

O sistema de fertirrigação consistiu de uma unidade central de controle (sistema de bombeamento, filtros de areia e tela, injetor de fertilizantes, manômetros e conexões), linha principal de tubos PVC, PN80, linhas de derivação de PVC, PN 40, linhas laterais com tubo flexível de polietileno, PN 40, gotejadores e registros. Utilizou-se um injetor de fertilizantes TMB modelo WP10, (Amiad Filtration Systems Ltd., D.N. Galil Elyon, Israel) e cerca de $55 \mathrm{~L}$ por hora de calda foram injetados com $907 \mathrm{~L}$ por hora de água. A condutividade elétrica da água de irrigação variou entre $3,49 \mathrm{mS} \mathrm{cm}^{-1}$ $\left(0,10 \mathrm{~kg} \mathrm{~L}^{-1}\right.$ de adubo) e $12 \mathrm{mS} \mathrm{cm} \mathrm{cm}^{-1}\left(0,35 \mathrm{~kg} \mathrm{~L}^{-1} \mathrm{de}\right.$ adubo), conforme a quantidade de adubo dissolvido na calda. A condutividade elétrica do extrato saturado do solo, em P4 e P12, variou entre 0,20 e $0,52 \mathrm{mS} \mathrm{cm}^{-1}$, nas profundidades de 20, 50 e $90 \mathrm{~cm}$. Os gotejadores Katiff, de vazão de 3,8 L por hora (Hubel Irrigation Systems, Salvaterra de Magos, Portugal), foram espaçados em $30 \mathrm{~cm}$ na linha, para formar uma faixa molhada de $60 \mathrm{~cm}$ de largura, ao longo da fileira de plantas.

O controle da irrigação foi feito por meio de tensiômetros, com tensímetro de punção digital e escala de leituras em bar. Os tensiômetros foram instalados nas profundidades 0,$10 ; 0,25 ; 0,40$ e $0,60 \mathrm{~m}$. As irrigações foram efetuadas em turnos de rega fixos de duas vezes por semana, quando a tensão da água no solo atingiu valores próximos a $20 \mathrm{kPa}$. Para o cálculo da lâmina a ser aplicada, em 2007, foi considerada a média das leituras dos tensiômetros até $0,25 \mathrm{~m}$ e, após o desenvolvimento das plantas (2008 e 2009), a profundidade foi alterada para $0,6 \mathrm{~m}$ de profundidade. Os dados meteorológicos diários de precipitação e temperatura média do ar foram monitorados por estação meteorológica automática ( $\mu$ Metos, Sentek, Agrotecnologia, Ponte Nova, MG, Brasil), instalada na área do experimento (Figura 1).

A cultivar utilizada foi a Catiguá MG-3 (Catuaí Amarelo IAC 86 x Híbrido de Timor UFV 440-10), que tem como características: copa cônica; ramos produtivos de internódios curtos, com moderada ramificação secundária; frutos de coloração vermelha e ciclo de maturação médio; folhas novas de coloração bronze; elevada capacidade produtiva; e alta tolerância à ferrugem e ao nematoide-das-galhas da espécie Meloidogyne exígua (Oliveira \& Pereira, 2008). O plantio foi realizado em abril de 2007, no espaçamento de 2,5x0,6 m (6.666 plantas ha- $\left.{ }^{-1}\right)$. As mudas foram obtidas por propagação seminal, e cultivadas em pé-franco com uma haste ortotrópica.

As características avaliadas foram: número de ramos plagiotrópicos primários (NRA); número de

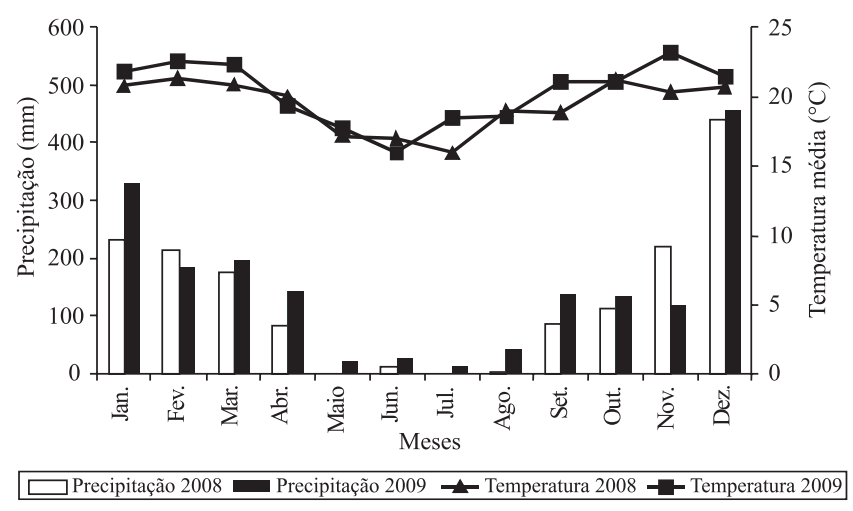

Figura 1. Precipitação $(\mathrm{mm})$ e temperatura média do $\operatorname{ar}\left({ }^{\circ} \mathrm{C}\right)$, de janeiro de 2008 a dezembro de 2009, em Lavras, MG. 
nós no ramo plagiotrópico primário $(\mathrm{NNO})$, obtido pela média de nós em ramos marcados nos dois lados da planta; altura da planta (ALT) e diâmetro de copa (DCO), obtidos em centímetros, com régua graduada; e diâmetro do caule (DCA), mensurado em milímetros com uso de paquímetro. No primeiro ano, as avaliações de crescimento foram realizadas em seis épocas, ao final de janeiro, março, maio, julho, setembro e novembro de 2008. No segundo ano, as avaliações foram realizadas em quatro épocas: janeiro, maio, setembro e novembro de 2009. Previamente às análises, os dados originais foram submetidos aos testes de normalidade de resíduos, de Shapiro-Wilk, e de homogeneidade das variâncias, de Bartlett, para então serem realizadas as análises individuais e conjuntas.

A comparação entre os dois experimentos, P4 e P12, foi realizada por análise conjunta, um mês após a última aplicação do P12 (novembro/2008 e novembro/2009). O efeito de doses foi estudado em análise de parcela subdividida no tempo, específica para cada experimento, tendo-se considerado todas as épocas avaliadas. Efetuou-se a análise de variância dos dados à significância de 5\% de probabilidade pelo teste F, com uso do SAS (SAS Institute, 2003).

\section{Resultados e Discussão}

Para o cafeeiro cultivado em sequeiro, a adubação pode ser dividida em adubação de primeiro e de segundo ano de formação da lavoura (Guimarães et al., 1999). Os resultados foram apresentados e discutidos conforme essa classificação.

No primeiro ano de formação da lavoura, não houve interação entre doses e épocas de avaliação no parcelamento em 4 e 12 aplicações de adubo, para todas as características estudadas. Para melhor discussão dos resultados, apenas o fator dose foi considerado no presente trabalho. Constatou-se, em ambos os parcelamentos, que o incremento ou a redução na dose-padrão de $\mathrm{N} \mathrm{e}_{2} \mathrm{O}$, recomendada por Guimarães et al. (1999) para o cultivo em sequeiro (100\%), não promoveu alterações no crescimento vegetativo do cafeeiro durante o primeiro ano de formação. Portanto, seja no P4 ou no P12, o aumento de $90 \%$ ou a redução de $30 \%$ da dose recomendada de $\mathrm{N}_{\text {e }} \mathrm{K}_{2} \mathrm{O}$ não alterou o crescimento vegetativo do cafeeiro.

Em relação à altura de planta, o crescimento em valores absolutos entre as doses variou de 38,8 a
$41,0 \mathrm{~cm}$, sem diferença significativa. Pequena variação em valores absolutos também foi observada nas características NRA (13,2 a 15,3 ramos), NNO (7,8 a 8,9 nós por ramo), DCA $(0,9$ a $1,0 \mathrm{~cm})$ e DCO $(43,4$ a $48,9 \mathrm{~cm})$, sem diferenças significativas entre esses. Para ALT, o crescimento oscilou entre 40,9 e 41,9 cm; para NRA, entre 15,3 e 15,5 ramos; para NNO, entre 7,7 e 8,4 nós por ramo; para DCA, entre 1,0 e 1,1 cm; e para DCO, entre 51,3 e $53,6 \mathrm{~cm}$.

Como as doses não apresentaram efeito significativo sobre o crescimento vegetativo do cafeeiro no primeiro ano, o efeito do fator parcelamento foi desconsiderado na discussão relativa aos resultados encontrados para o efeito de doses.

Vários fatores podem condicionar a falta de resposta e a suficiência da dose inferior $(70 \%)$, para o que se refere ao crescimento vegetativo do cafeeiro. Os teores de matéria orgânica (MO) $\left(4,3\right.$ dag $\left.\mathrm{kg}^{-1}\right)$ e de $\mathrm{K}\left(137,0 \mathrm{mg} \mathrm{dm}^{-3}\right)$ existentes no solo, previamente à implantação dos tratamentos, podem explicar parcialmente a ausência de resposta às doses de $\mathrm{N}$ e $\mathrm{K}$. Contudo, considerando-se que as doses aplicadas foram baseadas na recomendação existente para o cultivo em sequeiro, e que a adubação nessa forma de cultivo já foi amplamente estudada ao longo de muitos anos, acredita-se que outros fatores tenham maior participação na economia de adubos observada neste trabalho para o cultivo fertirrigado.

Se considerarmos a condição de fertilidade inicial do solo como preponderante na suficiência da dose inferior de $\mathrm{Ne} \mathrm{K}(70 \%)$, o efeito do fator parcelamento seria sensivelmente reduzido, uma vez que o P4 e o $\mathrm{P} 12$, por serem irrigados o ano todo, têm condições favoráveis à mineralização da $\mathrm{MO}$ e à absorção de $\mathrm{K}$. Assim, seria de se esperar semelhança entre as formas de parcelamento, fato que não foi observado.

É provável que com o adensamento do cultivo (densidades maiores do que 5.000 plantas por hectare), as recomendações de adubação por cova estejam superestimando a demanda das plantas, pois, com a redução no espaço entre plantas na linha de cultivo, geralmente menores que $1 \mathrm{~m}$, há a tendência de se aumentar a competição entre plantas por fatores do ambiente, como luz e $\mathrm{CO}_{2}$, o que causa redução na produção de biomassa vegetativa por planta e consequente diminuição da demanda de nutrientes por planta (Figueiredo et al., 2006; DaMatta et al., 2007). Embora seja esperado maior consumo de nutrientes por 
área, com o adensamento, o volume de solo explorado pelas raízes, sobretudo na fase de formação, aumenta consideravelmente, inclusive em profundidade, e pode reduzir as perdas de $\mathrm{N}$ e $\mathrm{K}$ por lixiviação e de N por volatilização (Braccini et al., 2002; Fenilli et al., 2007).

$\mathrm{Na}$ fase produtiva da cultura, Figueiredo et al. (2006) estudaram doses de nutrientes por planta para o cultivo adensado em sequeiro, em relação à dose recomendada por Guimarães et al. (1999), e relataram reduções de $57 \%$ quanto ao $\mathrm{N}$ e $44 \%$ quanto ao $\mathrm{K}$. Ainda quanto à fase produtiva da cultura, Pavan et al. (1994) estudaram doses de NPK e densidades de plantio do cafeeiro em sequeiro e relataram, quanto ao cultivo adensado, a redução de $50 \%$ na dose de NPK por planta em relação à utilizada no cultivo convencional.

Considerando-se que a redução na dose também foi indicada em estudos da fase produtiva do cafeeiro, em que as doses de $\mathrm{N}$ e $\mathrm{K}$ propostas por Guimarães et al. (1999) foram calculadas por área, pode-se inferir que o cultivo adensado aumenta a eficiência de utilização dos fertilizantes aplicados, o que justifica a redução nas doses de $\mathrm{N}$ e $\mathrm{K}$.

Uma vez que a época de adubação do cafeeiro em sequeiro coincide com temperaturas e precipitações elevadas (Figura 1), e que cada planta possui capacidade limitada de absorção no tempo, é provável que Guimarães et al. (1999) tenham recomendado doses superiores à demanda das plantas, por considerarem elevadas as perdas de $\mathrm{N}$ e $\mathrm{K}$.

Segundo Malavolta (1993), no primeiro ano de formação do cafeeiro (18 meses, cultivar Catuaí, 1.250 covas ha ${ }^{-1}$ ), o acúmulo de $\mathrm{N}$ e $\mathrm{K}$ na massa de matéria seca total (1,3 $\mathrm{kg}$ por cova) é de $28,7 \mathrm{~g}$ de $\mathrm{N}$ e $20 \mathrm{~g}$ de $\mathrm{K}$ por cova, valores bem inferiores ao da fase produtiva (42 meses), em que o acúmulo é de 143,5 g de $\mathrm{N}$ e 130,2 g de K por cova. A diferença no acúmulo de nutrientes entre as fases de crescimento indica baixa demanda por $\mathrm{N}$ e $\mathrm{K}$ na fase vegetativa, e alta demanda a partir da fase produtiva (Malavolta et al., 2002; Laviola et al., 2007). Magalhães et al. (1987), em experimento conduzido por três anos agrícolas (1982/1983 a 1984/1985), não encontraram resposta à aplicação de $\mathrm{N}, \mathrm{P}$ e K nos dois primeiros anos após o plantio da lavoura. No entanto, no terceiro ano agrícola, houve resposta linear para $\mathrm{Ne}_{2} \mathrm{O}$.

Dessa forma, é evidente que a resposta ao aumento das doses está condicionada à idade fenológica da cultura. Isso demonstra a impossibilidade de se generalizar conclusões obtidas na fase de formação, para a fase produtiva da lavoura. Segundo Clemente et al. (2008), a dose ideal para o primeiro ano de formação da lavoura está entre 71 e $112 \%$ da adubação-padrão recomendada para macronutrientes, o que está de acordo com os resultados obtidos no presente trabalho para o primeiro ano, no cultivo fertirrigado.

Em trabalhos com cafeeiros irrigados (Winston et al., 1992; Nazareno et al., 2003), o aumento nas doses de $\mathrm{N}$ e $\mathrm{K}_{2} \mathrm{O}$ não tem resultado em maior desenvolvimento das plantas, na fase inicial e produtiva do cultivo, tendo-se observado, inclusive, possíveis reduções. É provável que a maior umidade do solo nos sistemas irrigados aumente a eficiência na nutrição desses cultivos, pois, segundo Marschner (1995), a adequada disponibilidade de água no solo favorece amplamente a absorção de nutrientes e aumenta diretamente o fluxo de massa ( $\mathrm{N}$ e $\mathrm{K})$ e difusão $(\mathrm{K})$ de solutos até a raiz.

Redução na dose recomendada também foi relatada em trabalhos com adubação líquida, para cultivos em sequeiro. Fagundes (2006) mostrou que, para a fase de cobertura pós-plantio, a adubação via líquida permite reduzir em $50 \%$ a dose proposta por Guimarães et al. (1999).

Rezende et al. (2010) avaliaram diferentes doses de NPK para cafeeiros fertirrigados em fase de formação, com uso da dose-padrão proposta por Matiello et al. (2005) para o cultivo em sequeiro, e não puderam constatar uma tendência comum quanto à dose mais adequada ao cultivo fertirrigado.

Considerando-se que o presente trabalho foi realizado em cultivo adensado (6.666 plantas ha ${ }^{-1}$ ), com índice de umidade do solo mantido pela irrigação sempre próximo ao da capacidade de campo, e que os adubos foram aplicados pela via líquida (fertirrigação), todos os fatores discutidos até aqui estiveram presentes. Assim, os resultados encontrados no presente trabalho corroboram os da literatura citada e justificam a ausência de resposta às doses superiores (130, 160 e 190\%) e a suficiência quanto ao desenvolvimento vegetativo na menor dose (70\%). Portanto, é possível que uma dose menor do que a testada neste trabalho possa atender ao crescimento de lavouras fertirrigadas no primeiro ano pós-plantio.

Os resultados permitem inferir que, em lavouras fertirrigadas sob cultivo adensado, pode-se reduzir em até $30 \%$ a dose de $\mathrm{N}$ e $\mathrm{K}_{2} \mathrm{O}$ recomendada por Guimarães et al. (1999) para lavouras de sequeiro em fase de 
formação. Como em plantios adensados a eficiência da adubação via fertirrigação é elevada, parece mais adequado que a recomendação de adubação para a fase de formação seja feita por área, assim como é realizado para a fase produtiva.

De acordo com a análise conjunta dos experimentos (P4 e P12), não houve interação entre doses e formas de parcelamento da adubação, em todas as características estudadas, o que permitiu o estudo isolado do fator parcelamento (Tabela 2).

Para todas as características avaliadas, o parcelamento em 12 aplicações foi superior ao parcelamento em quatro aplicações. Pode-se dizer que no P12 o ganho em relação ao P4 variou de 10 a 20\%, para as características ALT, NRA, NNO e DCA. Para o DCO, o ganho foi próximo a $30 \%$, o que mostra que o crescimento de ramos foi bem superior com o uso do parcelamento em maior número de aplicações.

Em relação à testemunha em sequeiro, o $\mathrm{P} 4$ foi superior apenas quanto a DCA. Para as demais características, não houve diferença significativa. Esse resultado difere do observado por Nazareno et al. (2003), que demonstrou efeito positivo da irrigação no crescimento da parte aérea de cafeeiro cultivado no Cerrado mineiro. Tal discordância mostra que, quando a distribuição de chuvas é adequada, conforme ocorreu no sul de Minas no ano em estudo (Figura 1), a fertirrigação em quatro parcelamentos apresenta vantagem limitada ao cafeicultor. Todavia, para um crescimento semelhante ao do cultivo em sequeiro, a fertirrigação possibilitou redução de $30 \%$ na dose de $\mathrm{N} \mathrm{e} \mathrm{K}_{2} \mathrm{O}$.

No P12, o crescimento foi superior em relação ao tratamento controle, em todas as características.
O P12 foi superior à testemunha em cerca de $25 \%$ para ALT, $14 \%$ para NRA, $19 \%$ para NNO e $52 \%$ para DCA e DCO.

Neste trabalho não foi observada produção de frutos considerável ao fim do segundo ano de formação, em virtude do plantio tardio (mês de abril).

Ao final do segundo ano de formação, não houve interação entre doses e épocas de avaliação no parcelamento em 4 e 12 aplicações de adubo, em todas as características estudadas. No P4, não houve diferença entre as doses, em todas as características avaliadas. No P12, não houve diferença entre as doses quanto à ALT, NRA e NNO. Para DCA e DCO, as doses de 70,100, 130 e 160\% foram semelhantes, e a dose de $190 \%$ proporcionou crescimento inferior. Tal fato indicou excesso de $\mathrm{N}$ e $\mathrm{K}$, o que possivelmente comprometeu o equilíbrio nutricional das plantas.

Não houve interação entre forma de parcelamento e doses, quanto às características de crescimento estudadas (Tabela 2). O parcelamento em doze aplicações foi superior ao parcelamento em quatro, quanto a ALT, NRA, NNO e DCO, e apresentou comportamento semelhante para DCA. Pode-se dizer que no P12 o ganho em relação ao P4 foi de cerca de 6 a 10\% para as características ALT, NRA, NNO e DCO.

Em relação à testemunha de sequeiro, o $\mathrm{P} 4$ foi superior apenas quanto ao DCA, tendo crescido cerca de $0,4 \mathrm{~cm}$ a mais que a testemunha. Como o P4 e o cultivo em sequeiro receberam o mesmo parcelamento da adubação, a única diferença observada pode ser atribuída à irrigação. Segundo Tausend et al. (2000) e DaMatta \& Ramalho (2006), o cafeeiro em sequeiro, para aumentar a eficiência no uso da água, aloca mais assimilados no crescimento de raizes e limita

Tabela 2. Médias da altura de planta (ALT, cm), do número de ramos plagiotrópicos (NRA), do número de nós por ramo plagiotrópico $(\mathrm{NNO})$, do diâmetro do caule $(\mathrm{DCA}, \mathrm{cm})$ e do diâmetro de copa (DCO, cm) dos cafeeiros, ao final do primeiro e do segundo ano, respectivamente, novembro de 2008 e 2009 , de acordo com os parcelamentos de $\mathrm{N}$ e $\mathrm{K}_{2} \mathrm{O}$, nas parcelas fertirrigadas e no cultivo convencional em sequeiro (controle).

\begin{tabular}{|c|c|c|c|c|c|c|c|c|c|c|}
\hline \multirow[t]{2}{*}{ Tratamento $^{(1)}$} & \multicolumn{5}{|c|}{ Final do primeiro ano: novembro 2008} & \multicolumn{5}{|c|}{ Final do segundo ano: novembro 2009} \\
\hline & ALT & NRA & NNO & DCA & DCO & ALT & NRA & NNO & DCA & DCO \\
\hline & \multicolumn{10}{|c|}{ Parcelas fertirrigadas $^{(2)}$} \\
\hline P4 & $56,65 b$ & $20,87 b$ & $10,78 b$ & $1,54 b$ & $62,55 b$ & $98,31 \mathrm{~b}$ & $44,83 b$ & $22,05 b$ & $3,40 \mathrm{a}$ & $133,63 b$ \\
\hline \multirow[t]{2}{*}{$\mathrm{P} 12$} & $60,58 \mathrm{a}$ & $23,77 \mathrm{a}$ & $12,90 \mathrm{a}$ & $1,72 \mathrm{a}$ & $80,88 \mathrm{a}$ & $104,48 \mathrm{a}$ & $48,33 \mathrm{a}$ & $23,86 \mathrm{a}$ & $3,50 \mathrm{a}$ & $144,95 \mathrm{a}$ \\
\hline & \multicolumn{10}{|c|}{ Cultivo em sequeiro } \\
\hline Controle P4 & $51,32^{\text {ns }}$ & $19,84^{\text {ns }}$ & $10,71^{\mathrm{ns}}$ & $1,20 *$ & $54,24^{\mathrm{ns}}$ & $92,85^{\text {ns }}$ & $41,50^{\mathrm{ns}}$ & $21,45^{\text {ns }}$ & $3,03 * *$ & $125,82^{\mathrm{ns}}$ \\
\hline Controle P12 & $48,41 *$ & $20,92 *$ & $10,87 *$ & $1,13 *$ & $53,13 *$ & $91,03 * *$ & $43,33 * *$ & $22,64^{\mathrm{ns}}$ & $3,04 * *$ & $119,89 * *$ \\
\hline
\end{tabular}

(1)P4, parcelamento da fertilização em quatro aplicações; P12, parcelamento em 12 aplicações; Controle, cultivo em sequeiro com parcelamento da adubação em quatro aplicações durante os meses de chuva, tanto em P4 quanto em P12. ${ }^{(2)}$ Médias seguidas de letras iguais nas colunas não diferem pelo teste F, a 5\%

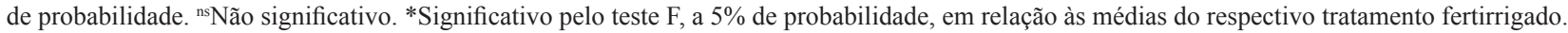


o crescimento do caule (calibre xilemático), para aumentar a absorção de água do solo e a resistência ao fluxo de água para a transpiração .

No P12, o crescimento foi superior em relação ao da testemunha em ALT, NRA, DCA e DCO. Destacase que a superioridade do $\mathrm{P} 12$ em relação ao cultivo convencional ocorreu em anos (2008 e 2009) em que o volume e distribuição de chuvas foram adequados para o cultivo de café não irrigado (Figura 1). Portanto, em anos atípicos ou na presença de veranicos em fases críticas da cultura, a superioridade do cultivo fertirrigado, sobretudo em 12 parcelamentos, deverá ser ainda maior. Tal fato justifica o uso da fertirrigação em regiões como o sul de Minas, consideradas aptas para o cultivo do cafeeiro sem a irrigação.

\section{Conclusões}

1. O parcelamento em 12 aplicações de $\mathrm{N}$ e K, via fertirrigação por gotejamento, é mais adequado do que o realizado em quatro aplicações, quanto ao crescimento do cafeeiro 'Catiguá' em plantio adensado, nos dois primeiros anos pós-plantio.

2. Para cafeeiros fertirrigados por gotejamento na fase de formação, em plantio adensado, pode-se reduzir em $30 \%$ a dose de $\mathrm{N}$ e $\mathrm{K}_{2} \mathrm{O}$ recomendada para o cultivo em sequeiro.

3. O crescimento do cafeeiro fertirrigado é superior ao do cultivado em sequeiro.

\section{Referências}

BRACCINI, M. do C.L.; BRACCINI, A. de L.; SCAPIM, C.A.; VIDIGAL FILHO, P.S.; ZABINI, A.V. Produção de grãos, concentração e aproveitamento de nutrientes em resposta ao aumento na densidade de plantio do cafeeiro. Acta Scientiarum, v.24, p.1205-1211, 2002.

CARVALHO, A.M. de; MENDES, A.N.G.; CARVALHO, G.R.; BOTELHO, C.E.; GONÇALVES, F.M.A.; FERREIRA, A.D. Correlação entre crescimento e produtividade de cultivares de café em diferentes regiões de Minas Gerais, Brasil. Pesquisa Agropecuária Brasileira, v.45, p.269-275, 2010.

CLEMENTE, F.M.V.T.; CARVALHO, J.G. de; GUIMARÃES, R.J.; MENDES, A.N.G. Faixas críticas de teores foliares de macronutrientes no cafeeiro em pós-plantio - primeiro ano. Coffee Science, v.3, p.47-57, 2008.

COSTA, E.L. da. Fertirrigação nitrogenada por gotejamento em cafezal e sua influência em características químicas do solo. 2005. 79p. Tese (Doutorado) - Universidade Federal de Viçosa, Viçosa.
DAMATTA, F.M.; RAMALHO, J.D.C. Impacts of drought and temperature stress on coffee physiology and production: a review. Brazilian Journal of Plant Physiology, v.18, p.55-81, 2006.

DAMATTA, F.M.; RONCHI, C.P.; MAESTRI, M.; BARROS, R.S. Ecophysiology of coffee growth and production. Brazilian Journal of Plant Physiology, v.19, p.485-510, 2007.

FAGUNDES, A.V. Adubação líquida na implantação da lavoura cafeeira (Coffea arabica L.). 2006. 54p. Dissertação (Mestrado) Universidade Federal de Lavras, Lavras.

FENILLI, T.A.B.; REICHARDT, K.; DOURADO-NETO, D.; TRIVELIN, P.C.O.; FAVARIN, J.L.; COSTA, F.M.P. da; BACCHI, O.O.S. Growth, development, and fertilizer- ${ }^{15} \mathrm{~N}$ recovery by the coffee plant. Scientia Agricola, v.64, p.541-547, 2007.

FIGUEIREDO, F.C.; FURTINI NETO, A.E.; GUIMARÃES, P.T.G.; SILVA, E. de B.; BOTREL, P.P. Eficiência da adubação com NPK na produção de cafezais adensados na região sul de Minas Gerais. Coffee Science, v.1, p.135-142, 2006.

GOMES, N.M.; LIMA, L.A.; CUSTODIO, A.A. de P. Crescimento vegetativo e produtividade do cafeeiro irrigado no sul do Estado de Minas Gerais. Revista Brasileira de Engenharia Agrícola e Ambiental, v.11, p.564-570, 2007.

GUIMARÃES, P.T.G.; GARCIA, A.W.R.; ALVAREZ V., V.H.; PREZOTTI, L.C.; VIANA, A.S.; MIGUEL, A.E.; MALAVOLTA, E.; CORRÊA, J.B.; LOPES, A.S.; NOGUEIRA, F.D.; MONTEIRO, A.V.C. Cafeeiro. In: RIBEIRO, A.C.; GUIMARÃES, P.T.G.; ALVAREZ V., V.H. (Ed.). Recomendação para o uso de corretivos e fertilizantes em Minas Gerais: 5a aproximação. Viçosa: Comissão de Fertilidade do Solo do Estado de Minas Gerais, 1999. p.289-302.

KARASAWA, S.; FARIA, M.A. de; GUIMARAES, R.J. Influência da irrigação e do parcelamento de fertirrigação sobre a produtividade, rendimento e qualidade do café (Coffea arabica L. cv. Topázio MG-1190). Ciência e Agrotecnologia, p.1427-1438, 2002. Edição especial.

LAVIOLA, B.G.; MARTINEZ, H.E.P.; SALOMÃO, L.C.C.; CRUZ, C.D.; MENDONÇA, S.M.; PAULA NETO, A. Alocação de fotoassimilados em folhas e frutos de cafeeiro cultivado em duas altitudes. Pesquisa Agropecuária Brasileira, v.42, p.1521-1530, 2007.

MAGALHÃES，J.C.A.J.; SAMPAIO，J.B.R.; SILVA, J.E. da. Adubação de manutenção de cafezais em solos de Cerrado. In: EMBRAPA. Centro de Pesquisa Agropecuária dos Cerrados. Relatório técnico anual do Centro de Pesquisa Agropecuária dos Cerrados 1982-1985. Planaltina: Embrapa-CPAC, 1987. p.346-349.

MALAVOLTA, E. Nutrientes e nutrição mineral de plantas. São Paulo: Agronômica Ceres, 1993. 210p.

MALAVOLTA, E.; FAVARIN, J.L.; MALAVOLTA, M.; CABRAL, C.P.; HEINRICHS, R.; SILVEIRA, J.S.M. Repartição dos nutrientes nos ramos, folhas e flores do cafeeiro. Pesquisa Agropecuária Brasileira, v.37, p.1017-1022, 2002.

MARSCHNER, H. Mineral nutrition of higher plants. $2^{\text {nd }}$ ed. London: Academic, 1995. 889p. 
MATIELLO, J.B.; SANTINATO, R.; GARCIA, A.W.R.; ALMEIDA, S.R.; FERNANDES, D.R. Cultura de café no Brasil: novo manual de recomendações. 2.ed. rev. ampl. Rio de Janeiro: MAPA: Procafé, 2005. 438p.

NAZARENO, R.B.; OLIVEIRA, C.A.D. da S.; SANZONOWICZ, C.; SAMPAIO, J.B.R.; SILVA, J.C.P.D.; GUERRA, A.F. Crescimento inicial do cafeeiro 'Rubi' em resposta a doses de nitrogênio, fósforo e potássio e a regimes hídricos. Pesquisa Agropecuária Brasileira, v.38, p.903-910, 2003.

OLIVEIRA, A.C.B.; PEREIRA, A.A. Cultivares de café portadoras de fatores de resistência à ferrugem indicadas para plantio em Minas Gerais. Belo Horizonte: Empresa de Pesquisa Agropecuária de Minas Gerais, 2008. 7p. (Epamig. Circular técnica, 34).

PAVAN, M.A.; CHAVES, J.C.D. ; ANDROCIOLI FILHO, A. Produção de café em função da densidade de plantio, adubação e tratamento fitossanitário. Turrialba, v.44, p.227-231, 1994.

PEREIRA, M.G.; LOSS, A.; BEUTLER, S.J.; TORRES, J.L.R. Carbono, matéria orgânica leve e fósforo remanescente em diferentes sistemas de manejo do solo. Pesquisa Agropecuária Brasileira, v.45, p.508-514, 2010.

REZENDE, R.; HELBEL JÚNIOR, C.; SOUZA, R.S. de; ANTUNES, F.M.; FRIZZONE, J.A. Crescimento inicial de duas cultivares de cafeeiro em diferentes regimes hídricos e dosagens de fertirrigação. Engenharia Agrícola, v.30, p.447-458, 2010.

SAS INSTITUTE. SAS system for microsoft windows. Version 8.2. Cary: SAS Institute, 2003.

SILVA, A.C.; SILVA, A.M. da; COELHO, G.; REZENDE, F.C.; SATO, F.A. Produtividade e potencial hídrico foliar do cafeeiro Catuaí, em função da época de irrigação. Revista Brasileira de Engenharia Agrícola e Ambiental, v.12, p.21-25, 2008 a.

SILVA, A.L. da; FARIA, M.A. de; REIS, R.P. Viabilidade técnico-econômica do uso do sistema de irrigação por gotejamento na cultura do cafeeiro. Revista Brasileira de Engenharia Agrícola e Ambiental, v.7, p.37-44, 2003.

SILVA, C.A. da; TEODORO, R.E.F.; MELO, B. de. Produtividade e rendimento do cafeeiro submetido a lâminas de irrigação. Pesquisa Agropecuária Brasileira, v.43, p.387-394, 2008 b.

TAUSEND, P.C.; GOLDSTEIN, G.; MEINZER, F.C. Water utilization, plant hydraulic properties and xylem vulnerability in three contrasting coffee (Coffea arabica) cultivars. Tree Physiology, v.20, p.159-168, 2000.

WINSTON, E.C.; LITTLEMORE, J.; SCUDAMORE, S.P.; O'FARREL, P.J.; WIFFEN, D.; DOOGAN, V.J. Effect of nitrogen and potassium on growth and yield of coffee (Coffea arabica L.) in tropical Queensland. Australian Journal of Experimental Agriculture, v.32, p.217-224, 1992.

Recebido em 11 de agosto de 2010 e aprovado em 14 de dezembro de 2010 